\title{
Gobernanza en la administración pública Revisión teórica y propuesta conceptual
}

\section{Jesús Antonio Treviño}

\section{Cantú}

Facultad de Arquitectura, Universidad Autónoma de Nuevo León jtrevino41@hotmail.com

\section{Resumen}

El objetivo de este trabajo es la búsqueda de la definición del término gobernanza y, por extensión, de la gobernanza metropolitana (metropolítica) en la teoría de la administración pública. En esta búsqueda se encontró que hay varias definiciones de gobernanza que sólo tienen sentido desde sus distintos enfoques de la administración pública. Se concluye que una buena gobernanza, de cualquier manera como se le defina en administración pública, puede favorecer situaciones indeseables (paradoja de la circularidad de las intenciones). Este escrito explica esta posible contradicción ubicando a los enfoques revisados y al cuerpo conceptual propuesto en el marco del ejercicio y las fuentes de poder.

Palabras clave: gobernanza, metropolítica, administración pública, economía política. 


\title{
Governance in public administration. Theoretical review and a conceptual proposal
}

\begin{abstract}
The aim of this article is to search a definition of governance and, by extension, of metropolitan governance (metropolitics) in public administration theory. This searching finds that definitions of governance only make sense from the specific conceptual approach addressing them. The essay concludes that actions to promote good governance may generate negative situations (the circularity of intentions paradox). A conceptual body articulated and suggested in this paper explains this paradox by locating all public administration approaches reviewed within a framework of social practices and sources of power.
\end{abstract}

Keywords: Governance, Metropolitics, Public Administration, Political Economy.

\section{Introducción}

A partir de los años noventa, el concepto de gobernanza cobró interés cuando las agencias internacionales de ayuda reportaron que la mala, o la ausencia de, gobernanza era una barrera importante para el desarrollo económico de los países subdesarrollados. El uso constante de la palabra gobernanza se difundió entre distintas campos del conocimiento: administración pública y política pública, relaciones internacionales, Unión Europea, política comparativa, países (buena o mala gobernanza) y corporaciones. Rohdes (2007: 5) advirte que la palabra tiene distintos significados entre los distintos campos, encuentra razonable delimitar o enmarcar la definición de gobernanza en la administración pública. Los párrafos siguientes retoman esta misma postura y señalan que la variedad de significados no sólo existe entre distintos campos, sino que también abunda entre las distintas perspectivas dentro del campo de la administración pública.

En administración pública, en general, gobernanza significa dirigir la economía y la sociedad hacia algunas metas colectivas (Peters, 2007: 554). La tesis básica en mi revisión es que, independientemente de su historia, este significado de gobernanza se reescribe en cada uno de los distintos enfoques de la administración pública, incluyendo el marco conceptual que propongo al final de este artículo. El trabajo sustenta este argumento en cuatro partes. La primera parte presenta la emergencia de un "estado hueco" (hollow state) que sustituye su intervención di- 
recta y capacidad ejecutiva por la negociación o diplomacia; se argumenta que los retos del "estado hueco" y las definiciones de gobernanza pueden analizarse desde cuatro enfoques de la teoría de la administración pública. La segunda parte señala cada uno de estos cuatro enfoques. La tercera parte indica que los análisis anteriores se localizan en el nivel analítico de las prácticas sociales y necesitan ser vistos como parte de un marco conceptual que aborde simultáneamente el ejercicio y las fuentes de poder; por esta razón, se sugiere enmarcar el estudio de la gobernanza desde la economía política. La cuarta parte sostiene que los enfoques de la administración pública y el marco propuesto no compiten entre sí porque pertenecen a niveles analíticos complementarios; en esta última parte también señala y explica que la búsqueda de la buena gobernanza puede generar situaciones sociales antidemocráticas.

\section{Nuevos desafíos del “estado hueco“ (hollow state)}

No hay duda de que las reglas y las regulaciones, los programas y los procesos que guían la sociedad han experimentado un cambio dramático. Este cambio en los mecanismos que guían y regulan la sociedad gira alrededor de tres temas o principios unificadores sobre el papel de la administración pública y la definición de la gobernanza (Peters y Pierre, 1998; Denhardt y Denhardt, 2007: 84-86; Frederickson y Smith, 2003: 207-210; Arnouts y Arts, 2009):

- El estado es desplazado como el actor principal de la política pública (Strange, 1996). Este desplazamiento ocurre por las presiones globales sobre los gobiernos nacionales a través de los mercados internacionales de capital y de organizaciones supranacionales (empresas trasnacionales, Banco Mundial, Fondo Monetario Internacional, Organización de Libre Comercio, Green Peace).

- Emergencia de redes de actores sociales. Son lazos formales e informales entre el estado y otros actores sociales, tales como profesionistas, sindicatos y grandes empresas. Los actores sociales también desarrollan redes de interacción entre ellos que son ajenas al estado. Las redes no-estatales son capaces de diseñar y operar políticas que anteriormente eran administradas de manera jerárquica por el estado.

- Cambios en la relación sector público-sector privado. Predomina la corriente de la nueva gerencia pública (NGP) que propone la adopción de la administración y técnicas de distribución de los recursos con base en las reglas del mercado; mayor ingerencia del sector privado en la provisión de los servicios públicos. 
La subcontratación de los servicios públicos reducen el papel del estado como oferente directo de los bienes públicos. Un esfuerzo deliberado para "adelgazar" el gobierno y descentralizar sus funciones con el fin de que deje de ser actor central en la formulación de políticas.

El desplazamiento del estado como actor central de la política pública, la emergencia de redes sociales autosuficientes, los cambios recientes en la relación sector público-sector privado generan un 'estado hueco' (hollow state). H. Brinton Milward, Keith Provan y Barbara Else, basados en un concepto que describe la estrategia de las empresas trasnacionales en la época de la globalización (hollow corporation), introdujeron en la administración pública la expresión "estado hueco" para señalar que un gobierno sustituye la provisión directa de servicios por la subcontratación, privatización y desregulación de actividades (Terry 2006). Los gobiernos centrales transfieren su capacidad de mando y descentralización de los servicios a los gobiernos locales y, por extensión, a terceros (actores no lucrativos y sector privado). ${ }^{1}$ Esta sustitución de funciones replantea el diseño de mecanismos para la rendición de cuentas, la comunicación y coordinación de decisiones entre los distintos actores y la creación de nuevas herramientas intelectuales en la administración pública (Rhodes, 2007: 1248; Bevir, 2007a: 419).

\section{Enfoques de la teoría de la administración pública y definición de la gobernanza}

Los retos del "estado hueco" y las definiciones de gobernanza en general, y de la gobernanza metropolitana en particular, pueden analizarse desde cuatro enfoques de la teoría de la administración pública: ${ }^{2}$

\footnotetext{
${ }^{1}$ Algunos sinónimos del "estado hueco" (hollow state) en la literatura de la administración pública son "estado sombra" (shadow state), "gobierno por aproximaciones" (government by proxy), "gobierno de terceros" (thirdparty government), y "regímenes de contratación" (contracting regimes).

${ }^{2}$ Se escoge esta opción sugerida por Frederickson y Smith (2003) con la intención de tener a las teorías de la administración pública como un referente conceptual familiar. Sin embargo, una alternativa igualmente importante es seguir los cinco modelos de gobernanza propuestos por Pierre y Peters (2005): a) estatista, el estado es el actor principal y controla la participación de otros actores sociales; b) liberal-democrático, el estado es el actor principal bajo presión de otros actores sociales; c) centrado en el estado, todo gira en torno al estado, pero su relación con otros actores sociales está institucionalizada; d) escuela holandesa, el estado es un actor más en la red social de la gobernanza; y e) gobernanza sin gobierno, el estado pierde legitimidad y poder frente a otros actores sociales.
} 
1. Gobernanza como administración pública tradicional o progresiva ${ }^{3}$ (autores clásicos, antes de 1968). La gobernanza es el diseño e implementación de políticas dirigidas a objetivos específicos, políticamente definidos. Los funcionarios públicos canalizan estas políticas a través de las diversas dependencias de gobierno organizadas jerárquicamente y están sujetas a los principios marcados por la ley y la realidad política. El gobierno es el actor más importante en la dirección de la sociedad, sin que ello implique que otros intereses no sean representados. Los funcionarios públicos reportan a los líderes políticos elegidos democráticamente.

Problemas. La política pública es formulada y controlada por expertos que determinan la naturaleza del interés público y diseñan las políticas óptimas para alcanzarlo. Las personas son inducidas por un sistema de premios y castigos para lograr las metas de las políticas científicamente elaboradas. No hay participación ciudadana que integre directamente los deseos sociales en la política pública.

2. Gobernanza como nueva gerencia pública (NGP) (Kettl, 2002). La gobernanza es la NGP. La gobernanza es la adopción de los valores y prácticas empresariales en el sector público. Las dos variantes principales de la NGP son el modelo Westminster (Nueva Zelanda, Australia, Canadá y el Reino Unido) y el modelo de la reinvención del gobierno (EE.UU.). Este segundo enfoque considera que la suma de los intereses particulares genera un resultado congruente con el de los grupos mayores de ciudadanos (consumidores). Las vertientes teóricas principales de la NGP son la teoría gerencial racionalista (managerialism), la teoría de la acción racional para la maximización de utilidades (public-choice theory), la economía de los costos de transacción (transaction costs) y la teoría del agente principal (principal-agent).

Problemas. La noción de gobernanza en la NGP es demasiado reducida. En situaciones de corrupción y/o poca transparencia, tal como lo señala Stiglitz

\footnotetext{
3Pareciera contradictorio utilizar "tradicional" y "progresivo" como sinónimos. En los inicios de la administración pública norteamericana, el movimiento 'progresivo' de finales del siglo XIX e inicio del XX buscó la separación de la política y la administración con el fin de asegurar la eficiencia en la provisión de servicios y eliminar la corrupción burocrática. Estos principios del movimiento progresivo son el eje central de la administración pública tradicional que pasó de la gerencia científica de Taylor a la gerencia administrativa del POSDCORB (Planning, Organizing, Staffing, Directing, Coordinating, Reporting and Budgeting). Otros nombres de la administración pública tradicional son "gerencia administrativa," " administración clásica" o "modelo burocrático racional."
} 
(2003) para el caso de Rusia, la privatización que propone la NGP beneficia a unos pocos amigos del grupo en el poder. Además, la administración de los asuntos privados es muy distinta de la administración pública. Mientras en la administración pública se rinden cuentas a diversos grupos sociales bajo criterios sociales, en la administración privada se rinden cuentas a un propietario o grupo de accionistas asociados por el afán de lucro; por otro lado, los proveedores de servicios rinden cuentas a sus contratistas en vez de servir directamente al interés público. La filosofía y la práctica de la NGP crean instituciones administrativas frágiles que erosionan la confianza en el gobierno (Terry, 2006: 111) y su ideología cuestiona el papel cultural y filosófico de la democracia (Peters y Pierre, 1998).

3. Gobernanza como nuevo servicio público (NSP) (Denhardt y Denhardt, 2007). La gobernanza es el ejercicio de la autoridad pública. Es un concepto más amplio que el de gobierno. Son las tradiciones, instituciones y procesos que determinan el ejercicio del poder en una sociedad, incluyendo cómo se toman las decisiones en asuntos de interés público y cómo los ciudadanos participan en las decisiones públicas (Denhardt y Denhardt, 2007: 86). En esta modalidad de gobernanza los ciudadanos escogen los servicios públicos que desean, deciden las cantidades y definen el modo de provisión mediante mecanismos de participación ciudadana, tales como fondos, contratos, sociedades y convenios (Callahan, 2007: cap. 9). En el NSP el estado depende cada vez más de otras organizaciones para llevar a cabo sus metas, aplicar sus políticas y definir su estilo de gobierno. Este enfoque tiene como antecedente la nueva administración pública (no confundirla con la nueva gerencia pública previamente descrita), surgida después de la conferencia de Minnowbrook (NY, 1968), "refundada" por el Manifiesto de Blacksburg (VA, 1983) y revaluada por las conferencias de Minnowbrook II (1988) y III (2008). ${ }^{4}$

Problemas. El NSP sólo es real como planteamiento. En el mundo hay un continuum de gobernanzas que van desde el modelo estatista (el gobierno es el actor principal) hasta la "gobernanza sin gobierno" (los actores sociales son más importantes que el gobierno). Los modelos de gobernanza que se acercan al modelo ideal del NSP serían la escuela holandesa (el estado es uno de los muchos

${ }^{4}$ Los reportes de la conferencia reciente de Minnowbrook III (2008) no muestran avances significativos o tan importantes como los de la conferencia de Minnowbrook I (1968). 
actores que participan en las redes sociales de gobierno) y el de "gobernanza sin gobierno," ya mencionado (Pierre y Peters, 2005). La gobernanza del NSP es más democrática que en los enfoques anteriores porque intervienen más actores sociales dispersos en diferentes niveles en la toma de decisiones. Sin embargo, esta multiplicación o "sobrecarga" de actores no garantiza que se resuelvan los problemas y dificulta saber quién está a cargo o con quién realizar acuerdos generales (Arnouts y Arts, 2009: 210). Por esta razón, Peters (2007: 554) aclara que el incremento de la importancia de los actores no-gubernamentales no implica la desaparición del estado, sino una atenuación de las decisiones jerárquicas en la toma de las decisiones públicas.

4. Gobernanza como conjunción administrativa (CA) (Frederickson, 1999). La gobernanza es el intento de crear una imagen coherente de las relaciones laterales e interinstitucionales en la administración pública frente a los nuevos retos al nivel local:

- Límites jurisdiccionales porosos. Los problemas rebasan los límites municipales y los residentes tienen compromisos sociales, políticos y financieros en áreas diferentes a la que viven o trabajan.

- Erosión de la capacidad de las jurisdicciones para contener y administrar los problemas complejos sociales, económicos y políticos. Las ciudades más grandes tienen los problemas mayores y menor capacidad individual para resolverlos por sí mismas.

- Problemas para distinguir lo público de lo privado. Los servicios públicos se subcontratan cada vez más en algunas jurisdicciones.

- El liderazgo político es más importante al interior de los municipios que entre ellos.

La teoría de la conjunción administrativa — planteada originalmente a nivel metropolitano, gobernanza metropolitana o metropolítica en términos de Myron Orfield— destaca dos aspectos:

a) Los retos metropolitanos se parecen mucho a los que debe afrontar la diplomacia en el nivel internacional. Los sistemas o redes de cooperación interjurisdiccionales son como los de la diplomacia entre las naciones-estado: 
En la ausencia de una autoridad central, y bajo condiciones de gran interdependencia, hay sistemas de cooperación y acuerdo altamente desarrollados en áreas metropolitanas que sirven esencialmente al mismo propósito que las prácticas de la diplomacia entre naciones estado. Como en el caso de la diplomacia, los patrones de cooperación y acuerdo en la metrópolis varía según la materia de que se trate, el estatus e intereses de los actores, la permanencia o inestabilidad de los problemas y el nivel relativo de la formalidad del proceso y procedimiento. Frederickson (1999: 707)

b) Para la política, las jurisdicciones políticas todavía son importantes en el sentido de las campañas electorales; la política aún es jurisdiccional. Si las estructuras jurisdiccionales jerárquicas se consideran como edificios, la conjunción administrativa es como los puentes peatonales que los conectan; es decir, la conjunción administrativa necesita de las jurisdicciones como entidades políticas. La conjunción administrativa es el arreglo y carácter horizontal de la asociación formal e informal entre los actores que representan unidades, con una conducta pública y administrativa interconectada.

Para sustentar su teoría, Frederickson (1999) señala que los oficiales de alto rango en Kansas City dedican aproximadamente $15 \%$ de su tiempo a las actividades de conjunción o cooperación interjurisdiccional. Esta conjunción se basa en los valores, creencias y experiencia del servicio público profesional más que en la autoridad formal.

Actualmente, la conjunción administrativa, la asociación horizontal de redes de varios actores públicos y la conducta administrativa resultante de la red describen mejor la administración metropolitana en la arena urbana. Se crean colaboraciones metropolitanas en redes de sinergia (Meijers, 2007) que, como un todo, actúan como un régimen urbano de participantes interconectados con intereses mutuos y contradictorios (Meek, 2003: 929). Existe una conjunción metropolitana práctica porque las metrópolis deben funcionar como unidades coherentes y articuladas para competir en la economía mundial y capitalizar las ventajas de las aglomeraciones urbanas. Más aún, la naturaleza de los problemas son de índole intermunicipal: hay muchos problemas públicos, tales como la calidad del aire, el transporte, el uso del suelo, la protección civil o la seguridad pública, que rebasan los límites municipales y deben ser atendidos como parte de la gobernanza regional, ya sea formal o informal (Hall, 2005: 211-212). Como la política de los municipios me- 
tropolitanos es de naturaleza interna (i.e., campañas electorales), los acuerdos de cooperación intermunicipal generalmente son delegados a los profesionales o a los expertos sobre una base de conocimiento, no de autoridad formal. Como lo notara Holden (1964: 634):

Los burócratas municipales importantes son los oficiales con experiencia más o menos permanentes de las oficinas de gobierno y un juicio rápido muestra que la mayoría de los contactos intergubernamentales se lleva a cabo en este nivel (de director de finanzas a director de finanzas o de oficial de salud a oficial de salud).

La clave de la cooperación entre los municipios de una metrópoli es la naturaleza interjurisdiccional de sus problemas. No hay organizaciones e instituciones más interdependientes que las metropolitanas. No debería sorprender que las mejores formas de cooperación se encuentren en las áreas metropolitanas; aunque no hay dos áreas metropolitanas iguales, son relativamente similares en términos gubernamentales; por lo tanto, los hallazgos y puntos teóricos hechos para los estudios de caso son generalizables.

Problemas de la gobernanza como conjunción administrativa. La política de cualquier jurisdicción puede crear fuerzas opositoras a la cooperación; los funcionarios pueden tener fricciones por diferencias de personalidad, partido político o presupuesto; no hay un estudio satisfactorio en nivel regional o subnacional.

La gobernanza metropolitana como conjunción administrativa, por las características de operación de redes metropolitanas, pudiera considerarse más cercana al enfoque del nuevo servicio público que al de la nueva gerencia pública.

Los cuatro enfoques revisados hasta aquí, siguiendo los pasos metodológicos de Denhardt y Denhardt (2007) y Pierre y Peters (2005), pueden graficarse como un continuum desde la administración pública tradicional (APT), centrada en el estado, hasta el nuevo servicio público (NSP), caracterizado por redes sociales. La nueva gerencia pública (NGP) se localiza en un punto intermedio entre la APT y el NSP. En este continuum, algunos autores identifican nueve (Van Kersbergen y Van Waarden, 2004), siete (Rhodes, 2000) o cinco (Hirst, 2000; Pierre y Peters, 2005) definiciones distintas de gobernanza. Si esta profusión de significados no fuera suficiente, Arnouts y Arts (2009: 205) señalan que el desplazamiento de la gobernanza desde la administración pública tradicional hacia las redes el nuevo servicio pú- 
blico genera dos tipos de gobernanza: buena gobernanza, también conocida como positiva o democrática, y mala gobernanza, también llamada negativa (ver figura 1). Ninguna clasificación o taxonomía es definitiva. En algunas situaciones, las acciones para la buena gobernanza pueden llevar a la paradoja de la circularidad de las intenciones: ceteris paribus, la gobernanza legitima situaciones antidemocráticas o propicia su emergencia (Hewitt, 1998). Esta paradoja se explica en la última parte de este trabajo con un cuerpo conceptual articulado en la siguiente sección.

\section{Figura 1}

\section{Desplazamiento de la gobernanza en las teorías de la administración pública}

\begin{tabular}{|c|c|c|c|}
\hline Teoría & $\begin{array}{l}\text { Administración Pública } \\
\text { Tradicional (APT) }\end{array}$ & $\begin{array}{l}\text { Nueva Gerencia } \\
\text { Pública (NGP) }\end{array}$ & $\begin{array}{l}\text { Nuevo Servicio Público (NSP) } \\
\text { Conjunción Administrativa (CA) }\end{array}$ \\
\hline \multirow[t]{5}{*}{ Descripción } & $\begin{array}{l}\text { El estado es el actor principal } \\
\text { de la política pública }\end{array}$ & $\begin{array}{l}\text { El estado adopta los valores } \\
\text { y prácticas empresariales }\end{array}$ & $\begin{array}{l}\text { Redes de actores sociales } \\
\text { dominan la política pública }\end{array}$ \\
\hline & $\begin{array}{l}\text { Gobernanza } \\
\text { Vieja }\end{array}$ & & $\begin{array}{l}\text { Gobernanza } \\
\text { Nueva }\end{array}$ \\
\hline & & $\sqrt{ }$ & マ \\
\hline & & $\begin{array}{l}\text { Gobernanza } \\
\text { negativa }\end{array}$ & $\begin{array}{l}\text { Gobernanza positiva } \\
\text { (buena o democrática) }\end{array}$ \\
\hline & & $\begin{array}{l}\text { Redes con suficiente } \\
\text { control social para } \\
\text { resistir regulaciones e } \\
\text { imposiciones del } \\
\text { estado (i.e.., sindicatos } \\
\text { oNGOs). }\end{array}$ & $\begin{array}{l}\text { Caracterizada por variables } \\
\text { representativas de: } \\
\text { (1) voz y rendición de cuentas, } \\
\text { (2) estabilidad política y ausencia } \\
\text { de violencia, } \\
\text { (3) efectividad gubernamental, } \\
\text { (4) calidad regulatoria, } \\
\text { (5) estado de derecho, y } \\
\text { (6) control de la corrupción. }\end{array}$ \\
\hline
\end{tabular}

Fuente: Elaboración propia con conceptos e ideas de Denhardt y Denhardt (2007), Pierre y Peters (2005) y Arnouts y Arts (2009).

\section{Gobernanza y metropolítica en la economía política (EP)}

La gobernanza implica un conjunto de instituciones y procesos que tienen que ver con el ejercicio del poder en la sociedad. Las instituciones, en el sentido amplio, son patrones individuales o de grupo obligados por sanciones en leyes, costumbres o prácticas sociales informales (Bellah et al., 1991: 10-11, citado en Wamsley y Wolf, 1996: 29). El proceso de gobernanza se relaciona con la manera de tomar decisiones en una sociedad y cómo los ciudadanos y los grupos interactúan en la 
formulación de metas públicas y en la implementación de la política pública. Este proceso involucra muchos grupos y organizaciones diferentes con distintos grados de participación e interés (Denhardt y Baker, 2007: 131). En estas líneas no sólo interesa la definición de gobernanza sino también su ubicación en el nivel del ejercicio y fuentes de poder. Es importante subrayar también que se necesitan técnicas para el análisis de los procesos de negociación de los diversos grupos con niveles de interés y participación desigual.

La gobernanza, como ejercicio del poder institucionalizado en el nivel de las prácticas sociales, es importante en el proceso de estructuración porque es el proceso por el que se fortalecen las fuentes de poder que, a su vez, determinan el alcance de la acción (institucional o no institucional) de los actores sociales. Parafraseando a Giddens (1976: 161), la gobernanza es parte del proceso por el que los actores sociales ejercen su poder $\mathrm{y}$, en esa medida, robustecen —o debilitan- sus fuentes de poder. Esas fuentes de poder fortalecidas - o disminuidas - a su vez determinan las posibilidades de la acción social. De esta manera, las fuentes de poder son construidas por la acción social y la acción social, a su vez es determinada por las fuentes de poder (ver figura 2). Es obvio que en esta perspectiva la realidad social es una construcción dinámica con intereses específicos que guían acciones deliberadas o intencionadas.

En esta sección se aborda la gobernanza y la metropolítica (gobernanza metropolitana) en el marco de la economía política; por lo tanto, conviene desglosar a mayor detalle las ideas anteriores. La sección siguiente adapta a la gobernanza algunos desarrollos teórico-metodológicos de la economía política internacional (EPI). La idea clave que une estos dos temas es la gobernanza metropolitana como problema de diplomacia, no en el sentido sugerido por Holden (1964), sino en el de la "diplomacia triangular" en el ámbito de la EPI. En su síntesis de las teorías de la gobernanza, Frederickson y Smith (2003) redescubren la propuesta de Holden (1964) que considera a la gobernanza metropolitana como un problema de diplomacia. Después de revisar someramente el trabajo de Susan Strange (1996) sobre la pérdida de fuerza de los estados nacionales frente a las empresas trasnacionales y la permeabilidad de las fronteras nacionales a las fuerzas de la globalización (Retreat of the State), Frederickson y Smith (2003) limitan su análisis a la diplomacia en el sentido tradicional concluyendo que los problemas de la gobernanza metropolitana son resueltos por una "conjunción administrativa." Frederickson y su equipo dedican su obra reciente a medir el tiempo que los servidores públicos electos y los burócratas dedican a la conjunción administrativa en Kansas (1999 y 
2009) y a la tipología de la administración de las ciudades americanas (2004); es decir, Frederickson et al., tal como lo hiciera en su tiempo Holden para la diplomacia tradicional, se interesan en las lecciones que pueden aprender de los problemas y soluciones de cooperación interjurisdiccional. Con ello, sin embargo, Frederickson et al. omiten todo el desarrollo conceptual sobre "diplomacia triangular" de Susan Strange y su potencialidad para ser adaptado al ámbito metropolitano.

\section{Método Burke-Aristóteles en la "gramática de los motivos"}

Susan Strange (1988, 1991 y 1996) propone una serie de preguntas básicas para el estudio del sistema global similares a las que utiliza un antiguo método esbozado por Aristóteles (Poética y Retórica) y rescatado y mejorado por Kenneth Burke (1945) para el análisis del discurso.

Si quieres entender el mundo, tienes que hacerte estas preguntas: ¿Quién obtiene qué? ¿Por qué la estructura es de la manera que es? ¿Cuáles son las consecuencias? ¿Quién arriesga? ¿Quién tiene las oportunidades? ¿Quién paga? ¿Quién se beneficia? (Susan Strange, Entrevista personal, 1997).

El método de Burke-Aristóteles se conoce como "análisis pentáico" (pentadic analysis) por apoyarse en cinco preguntas: 1) ¿qué ocurrió?, 2) ¿cuándo o dónde pasó?, 3) ¿quién lo hizo?, 4) ¿cómo lo hizo? y 5) ¿porqué lo hizo? Estas interrogaciones se traducen en cinco categorías que definen cualquier acción humana: qué ocurrió (acto), cuándo y dónde pasó (escena), quién lo hizo (agente), cómo o de qué modo lo hizo (agencia), y por qué (propósito). Estas cinco preguntas comparten ciertas interrelaciones formales por ser atributos de una misma cosa o sustancia. Por la interrelación de estos elementos en la estructura de la acción, en lo que Burke llama la "gramática de los motivos," la respuesta a una de estas preguntas afecta a las restantes.

En el caso del sistema económico global, los principales actores son las empresas transnacionales y los estados nación; sus interrelaciones dan lugar a la llamada la "diplomacia triangular" (Strange y Stopford, 1991): relación empresa-empresa, de la que se ocupa la economía; relación estado-estado, materia de la diplomacia tradicional; y relación estado-empresa, considerada por la ciencia política y otras disciplinas. Es claro que el método de la diplomacia triangular no sólo identifica los principales actores de la escena global sino que, por omisión, clasifica al resto de los actores por su grado de participación, interés o lejanía en un caso especí- 
fico. Existen métodos complementarios que apoyan la clasificación y análisis de la diplomacia triangular, tales como "el método Gordon de rectángulos o círculos concéntricos" (Weiss, 1990; Gordon, 1977; Dekmejian y Simonian, 2003), la negociación por "áreas problema" (Brewer, 1992) o el "cubo de negociación" (Behrman y Grosse, 1990: Ch.1 y Ch. 8). Los enfoques complementarios sobre el interés y desempeño de actores diversos, primarios y secundarios, tienen el inconveniente de aplicarse al nivel de las negociaciones, en circunstancias cambiantes, sin referir explícitamente las fuentes de poder en operación. Los métodos para el estudio de las negociaciones entre los actores son importantes siempre que se les ubique en la dimensión adecuada, el de la negociación y acuerdos, no en la economía política metropolitana. Los métodos de rectángulos o círculos concéntricos, áreas problema o cubos de negociación pueden ser de gran utilidad si las relaciones entre los actores se remiten a sus fuentes de poder, tal como lo hace el análisis de las relaciones en la "diplomacia triangular".

Una tarea importante en el análisis de la gobernanza metropolitana es identificar los principales actores y analizar sus relaciones. Como no hay adaptaciones del método de Susan Strange en el nivel local se puede considerar, por ejemplo, que hay dos actores principales que forman una diplomacia triangular: gobiernos municipales y desarrolladores inmobiliarios. ${ }^{5}$

Economía política metropolitana: de la gramática de los motivos a la gramática de la acción social

El método de Burke-Aristóteles, originalmente apoyado en cinco preguntas: ¿qué ocurrió?, ¿cuándo o dónde pasó?, ¿quién lo hizo?, ¿cómo lo hizo? y ¿por qué lo hizo? puede extenderse a seis interrogantes agregando: ¿qué posibilidades tuvo?

De igual manera, a las cinco categorías respectivas (acto, escena, agente, agencia, propósito o motivo) se suma una sexta: fuentes de poder, es decir, ¿qué hace que los actos sean posibles?, ¿qué hace que las cosas sean como son? La pregunta

\footnotetext{
${ }^{5}$ Esto no significa que no haya otros grupos locales importantes, tales como grupos ecologistas, sindicatos, universidades. La identificación y multiplicación de los actores sociales considerados depende del problema específico. De hecho, Holden (1964), en su diplomacia metropolitana, y Frederickson y Smith (2003), en su conjunción administrativa, restringen la identificación de sus actores principales a los gobiernos municipales. En realidad, poco importa si la diplomacia es triangular (Strange y Stopford 1991), pentagonal (Lawton 1996) o poligonal. Lo relevante es considerar que las negociaciones de los actores sociales expresan y modifican sus fuentes de poder. Estas últimas, a su vez, determinan los temas de la agenda, el proceso y resultado final de las negociaciones.
} 
natural es, ¿por qué agregar una sexta interrogante? La respuesta directa es: para llevar la gramática de los motivos a un cuerpo coherente de teoría social. ${ }^{6}$ Un tema central en la literatura sobre el sistema global (Strange 1988 y Strange y Stopford 1991, principalmente) es el de las posibilidades de la acción (fuentes de poder). La introducción de la sexta pregunta, al llevar la gramática de los motivos al contexto del poder, desplaza la discusión del cómo se describen o comunican las cosas al por qué ocurren los hechos. Por el traslado de la descripción o comunicación a la explicación, las primeras líneas de esta sección muestran que las preguntas de Susan Stranage son similares, no iguales a las del método Burke-Aristóteles. En su Gramática de los motivos, Burke intuyó la economía política, pero no fue su interés principal:

Según Aristóteles, Tales creía que "todas las cosas están llenas de Dioses.” Para nuestros propósitos esto podría interpretarse como el reconocimiento del hecho de que en todo hay un poder, o motivo, de algún tipo (Burke, 1945: 118).

La incorporación de las fuentes de poder como determinantes de lo posible (¿qué posibilidades tuvo?). En los últimos veinte años, una parte importante de la literatura en la economía política internacional aborda el estudio del poder en dos dimensiones simultáneas (Strange, 1988, 1991 y 1996; Nye 1990, 2004 y 2008): la dimensión relacional ${ }^{7}$ (en el nivel de los actores o prácticas sociales) y la dimensión estructural (en el nivel de las fuentes de poder) (ver figura 2). Max Weber proporciona la definición más breve y conocida de la dimensión relacional: un actor social, A, tiene poder relacional si hace que otro actor social, B, haga algo que no hubiera hecho en otra circunstancia (Weber, 1957: 202-203). ${ }^{8}$ John W. Gardner (1990: 55) proporciona una definición similar para el poder relacional: es la capacidad de modificar intencionalmente la conducta de otros. La dimensión estructural es la que determina el resultado en la dimensión relacional. La dimensión estructural también es conocida como "bases" (French y Raven, 1959, en Shafritz

\footnotetext{
${ }^{6}$ Se pueden agregar todas las preguntas que sean, pero por distintas razones. Siguiendo la retórica de Aristóteles, se puede agregar una pregunta relativa a la actitud o al marco. El marco de una situación se refiere a la idea, ideología o intención implícita en la escena: puede ser una visión del mundo, patrón de comportamiento o razón que legitima la conducta de los actores. Un conjunto de marcos da el argumento de una narrativa; por ejemplo, los cuatro marcos de un argumento de "satanización" o "villanización" son: 1) identificar al oponentes, 2) exagerar el poder del enemigo, 3) mostrar al oponente en situaciones completamente negativas, y 4) mostrar que el oponente tiene motives perversos o corruptos. Generalmente este proceso se acompaña de otro de "santificación" del "bueno" por contraste al "malo."

${ }^{7}$ Después de todo, el poder sólo es concebido en relación con otro (s).

${ }^{8}$ Algunos autores atribuyen esta definición a Robert Dhal (i.e., Pfeffer, 1981, en Shafritz y Ott 1987: 310).
} 
y Ott, 1987), "fuentes" (Strange, 1988; Gardner 1990) o "recursos" (Nye, 2004 y 2008) de poder. Nye (2004 y 2008) proporciona la definición más clara de la dimensión estructural: hay sólo dos tipos de poder estructural: duro (hard) y blando (soft). Mientras el poder duro es la fuerza y la economía; el poder blando se refiere a los valores, el conocimiento las reglas y cualquier cosa que busque "atrapar la mente y el corazón de la gente" Aunque todos los tipos de poder duro y suave son interdependientes, algunos ejemplos representativos son Stalin o Hitler (poder duro-fuerza), Bill Gates o Carlos Slim (poder duro-economía) y el Papa (poder blando-valores):

El soft power es la capacidad de hacer creer a la gente que las ideas de uno son las mejores, que nuestros valores son los adecuados. Es el poder de moldear las ideas de la gente. Es más sutil que decir "si no me das tu dinero te mato," o "si no eres de esta manera te hago sufrir." Es sobre cómo comunicamos y qué comunicamos. Lo que comunicamos son creencias y sistemas o preferencias de valores (Susan Strange, Entrevista personal 1997).

En resumen. Se puede colocar en un librero de tres niveles la materia tratada en las seis preguntas anteriores: los agentes (actores) desarrollan acciones (actos o hechos) en una situación (escena), utilizando ciertos medios (agencia) para lograr sus fines (propósitos) posibles (fuentes de poder) (ver figura 2). Estos seis elementos son parte de un sistema interdependiente gobernado por relaciones históricas y dialécticas; históricas porque los actos del presente son consecuencia de las acciones de ayer; dialécticas porque los actos de hoy son resultado de la acción y reacción de los diversos agentes con intereses convergentes y/o divergentes en un momento determinado.

¿Economía política o gramática de la acción social? La gramática de los motivos de Burke, al agregarse un término sobre las posibilidades de la acción, se transforma en economía política más que en una gramática de la acción social ${ }^{10}$, por dos razones:

\footnotetext{
${ }^{9}$ Galbraith (1985) sugiere una clasificación parecida a la de Nye: Poder condigno (sumisión por la fuerza o el castigo), compensatorio (sumisión por retribución) y condicionado (sumisión voluntaria à la La Boétie: por convencimiento, persuasión, educación o compromiso social).

${ }^{10}$ Ésta es una expresión transitoria por motivos expositivos. Expresiones equivalentes a la gramática de la acción social serían la referida a la sociedad en general (gramática de las prácticas sociales) o a un ámbito específico (gramática del sistema global o gramática de la ciudad).
} 


\section{Figura 2}

\section{Librero de tres niveles para el estudio de la gobernanza}

1. Hechos. Crisis económica, deuda externa, empleo en empresas maquiladoras, dispersión urbana.

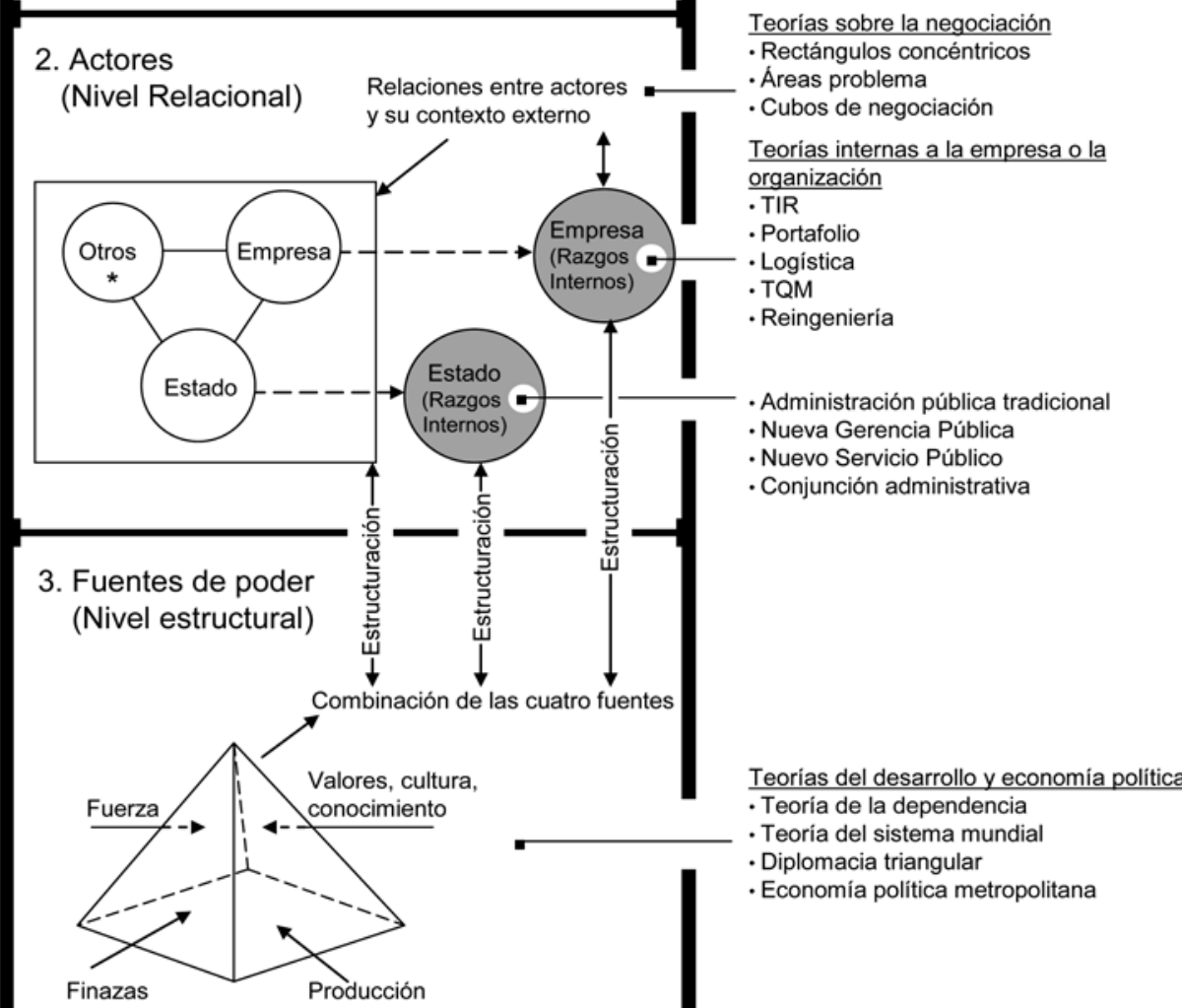

* "Otros" incluyen actores no-gubernamentales, empresas trasnacionales y otras empresas nacionales, sindicatos, organizaciones científicas y culturales, ambientalistas.

a) La introducción del tema del poder no sólo crea un cambio cuantitativo (en el número de preguntas), sino también cualitativo (en el enfoque del 
análisis). La economía política reinterpreta las cinco preguntas de BurkeAristóteles desde el ámbito de las fuentes de poder. La economía política no es la simple extensión de la gramática de los motivos. Al introducir el tema del poder en el sistema global o en la gobernanza metropolitana, la gramática de los motivos deja de ser gramática porque sustituye el interés por la forma con la explicación de las causas; abandona la retórica para convertirse en economía política (metropolitana, en el caso que nos ocupa). ${ }^{11}$

b) La preocupación en la economía política es analizar por qué ocurren las cosas; la preocupación en la gramática de los motivos es la forma en que se comunican los acontecimientos. Cuando el interés es analizar por qué ocurren los hechos en vez de cómo se reportan en la noticia, se abandona el análisis del discurso o de los medios para adentrarse en el estudio de los determinantes de la realidad social y sus procesos de estructuración. Al buscar los elementos determinantes de una retórica de acontecimientos (i.e., los reportes que se presentan en los medios de comunicación) nos alejamos de la gramática de los motivos para entrar al ámbito de la economía política.

Las dos razones anteriores muestran cómo la gramática de los motivos presta a la economía política sus cinco preguntas sin confundirse con ella. Teniendo esto muy claro, es posible usar con toda libertad las preguntas de la gramática de los motivos en la economía política. Una vez identificado el tema de interés, en la economía política hay tres tareas principales: la identificación de los principales actores, de sus relaciones más importantes y de sus fuentes de poder. La identificación de los actores más importantes filtra en el análisis la multiplicidad de hechos irrelevantes. La razón por la que deben identificarse las relaciones más importantes de los actores principales es la siguiente: un actor social, como un hombre singular, hace muchas cosas y no todas ellas son congruentes o articuladas entre sí. Por eso, conviene seleccionar las relaciones y los elementos que se consideren relevantes a la acción que se busca analizar.

\footnotetext{
${ }^{11}$ Es oportuno mencionar que economía política en estas notas refiere dos ideas principales: 1) la economía política no se restringe a la economía (producción y finanzas) como fuente de poder, también están la fuerza y el conocimiento, los valores, las ideas. Estas fuentes de poder remiten a las causas e impiden que la economía política sea considerada una mera gramática de la acción social, gramática de las prácticas sociales o gramática de la ciudad; y 2) la economía política fusiona dos términos que corresponden a distintos ámbitos analíticos: la economía, que corresponde al nivel de las fuentes de poder (de las posibilidades de la acción), y la política y las políticas de gobierno que se ubican en el nivel de los actores sociales (de la acción social). Esta fusión de términos en la expresión "economía política" permite una visión completa de la acción social y sus posibilidades, pero frecuentemente lleva a que el lector poco cuidadoso mezcle indistintamente los efectos con las causas (confusión de conceptos de la acción social con sus fuentes de poder).
} 
La economía política no permite la ambigüedad de términos que ocurre en la gramática de los motivos. Los actores son los únicos provistos - o carentes- de poder. Los casos donde un actor es sustituido por una escena o un medio por un actor no son aceptables. Esto no quiere decir que no haya análisis ingenuos o intencionados que incurran en esta práctica.

Si queremos mostrar las distintas versiones de un mismo hecho para ganar la voluntad de la audiencia, la gramática de los motivos es la más apropiada. Si queremos analizar los acontecimientos desde las fuerzas que les dieron origen, la economía política es la indicada. La segunda incluye a la primera y la descubre preguntando: ¿cuáles son los actores principales y cuáles sus relaciones mas importantes?, ¿qué posibilidades reales tienen esos actores de influir en otros?

\section{Nota final sobre la diplomacia triangular y los cuatro enfoques administrati- vos o gerenciales de la gobernanza metropolitana}

Las secciones anteriores muestran que la gobernanza tiene distinto significado, según el enfoque que la trate. Se muestra también que los cinco enfoques revisados buscan la buena gobernanza. En esta búsqueda, sin embargo, la consideración de las fuentes de poder marca una diferencia clara entre el enfoque propuesto y las cuatro perspectivas en la teoría de la administración pública revisadas en este trabajo. Enseguida se utiliza una metáfora basada en las ciencias naturales para ilustrar mejor esta distinción. Es oportuno recordar que la búsqueda del bien social - como quiera que se le defina - por medio de la gobernanza puede acarrear efectos de signo contrario. A esta posibilidad bien pudiera llamársele la "paradoja de la circularidad de las intenciones".

Diferencias en la idea de gobernanza en las teorías de la administración pública y la economía política ilustradas mediante la metáfora de la astronomía y la geología. La gobernanza en las teorías administrativas o gerenciales y en la economía política es tan diferente como el contenido de la teoría de la formación del universo y de la forma de los continentes. La teoría sobre el origen del universo, en la astronomía, responde a la pregunta sobre el inicio del universo; no responde a cómo se formaron los continentes o cómo tomaron forma. Esto último es materia de otra teoría, la geología. La economía política de la gobernanza metropolitana, como las teorías de formación del universo, considera a la ciudad en un contexto amplio: ¿por qué ocurren las cosas?, ¿quién se beneficia o perjudica? Por otro lado, las teorías administrativas y gerenciales de la gobernanza, como las teorías sobre 
la formación de los continentes aportan algo diferente: ¿cómo hacerlo?, ¿cómo manejar el carro?, ¿cómo administrar la ciudad? Lee el manual o aprende las lecciones de otros que lo han hecho mejor que tú. Son manuales de instrucciones para la "buena" administración de la ciudad, sin preocuparse por entenderla o explicarla. La economía política de la gobernanza, aunque más amplia en enfoque, no es mejor que la visión correspondiente en las teorías administrativas y gerenciales porque responden distintas preguntas. El reclamo de Denhardt y Denhardt (2007) de valorar a la gente sobre la productividad o la invitación de Frederickson (1999) de tomar a las áreas metropolitanas como el mejor referente empírico para el desarrollo de una teoría de la gobernanza basada en la conjunción administrativa son importantes en el nivel de las prácticas sociales. Estas prácticas, recordemos, se realizan a través de instituciones y procesos que representan la gobernanza en los enfoques administrativos o gerenciales. Los enfoques gerenciales, sin embargo, son de poca utilidad para explicar las consecuencias o las razones del ejercicio de poder en la sociedad.

Paradoja de la circularidad de las intenciones: ceteris paribus, la búsqueda de un bien propicia el efecto contrario. El Banco Mundial (BM) destaca entre los organismos internacionales que utilizan la palabra gobernanza para señalar una serie de fallas en los gobiernos nacionales sin pecar, al menos en apariencia, de intervencionistas. Para el BM, la gobernanza es el conjunto de tradiciones e instituciones por las que se ejerce la autoridad en un país. Esta definición, en un enfoque más cercano a la nueva gerencia pública que al nuevo servicio público, incluye el proceso de selección, monitoreo y reemplazo de los gobiernos, la capacidad del gobierno para formular e implementar políticas importantes, así como el respeto de los ciudadanos y del estado por las instituciones que regulan las interacciones económicas y sociales (Kaufmann, Kraay y Mastruzzi, 2009: 5). El redescubrimiento y adaptación de una sola palabra que combina todos estos elementos es una alternativa diplomática excelente para señalar eufemísticamente situaciones de corrupción o necesidades de reformas políticas y sociales. Sin embargo, las recomendaciones para mejorar la posición en los indicadores de la buena gobernanza deben tomarse con cautela porque pueden generar situaciones indeseables o contrarias a las esperadas (Hewitt, 1998). La buena gobernanza (o gobernanza democrática, como prefiriere denominarla Gore en su National Performance Review y Denhardt y Denhardt, 2007), se promueve fortaleciendo la sociedad civil. Este fortalecimiento es condición indispensable para lograr la descentralización y la administración participativa o participación ciudadana en la toma de decisiones (Maheshwari, 2002: 240-242). En muchos países subdesarrollados, sin embargo, el 
apoyo indiscriminado a la sociedad civil debilita en vez de fortalecer las bases de la democracia. La sociedad civil en los países pobres la constituyen organizaciones sociales que existen como estrategia de sobrevivencia. Sería absurdo suponer que los incentivos a estas organizaciones, aunque buenos para mitigar el hambre, llevan a una buena gobernanza. En otros casos, especialmente en países con guerra civil reciente, la sociedad civil es un grupo muy heterogéneo entre los que se cuentan los traficantes de armas y drogas, torturadores y personas acostumbradas a la violencia o dominadas por el odio étnico (Hewitt, 1998).

Además de las situaciones anteriores, en el espíritu de la economía política metropolitana en este artículo, la evaluación de términos que implican acción, tales como gobernanza, revolución o democracia, debe remitirse tarde o temprano a los resultados. Los individuos convocados en nombre del valor normativo de un concepto debieran correr a esconderse porque no sólo no podrán negarse al llamado, sino que cualquier indicio de resistencia o rebeldía se convierte en falta capital. ¿Quién osaría resistirse a la petición de la defensa de la revolución o al fortalecimiento de la democracia y sus instituciones? Sin embargo, si los conceptos normativos fueran revisados por los resultados, la verdadera naturaleza de la convocatoria queda al descubierto: revolución (escoja cualquiera) sería el movimiento armado donde la mayoría se mata entre sí para el beneficio de la minoría. Del mismo modo, democracia (escoja cualquiera) sería el gobierno donde las minorías hacen creer a la mayoría que ella es la que gobierna; de no ser así, por la magnitud e intensidad de la pobreza en muchos países democráticos, tendríamos que redefinir a la democracia como el gobierno donde la mayoría gobierna contra sí misma y para el beneficio de la minoría. ${ }^{12}$ Generalmente, se convoca a los individuos en nombre de un valor normativo o social para el beneficio particular o minoritario. Lo que interesa resaltar, en todo caso, es la existencia de una literatura importante que mide e incentiva la buena gobernanza o la gobernanza democrática por las condiciones favorables o adversas para la participación de la mayoría, independientemente de la apropiación relativa de los beneficios. Esta actitud puede llevar a la paradoja de que la búsqueda de la buena gobernanza sea el resultado de situaciones antidemocráticas que intentan legitimarse o, en otros casos, provoque y oculte esas situaciones. Esta paradoja es fácil de explicar en el marco de la eco-

\footnotetext{
${ }^{12}$ El gobierno de la minoría con investidura de mayoría no es la única distorsión de la democracia. También existe el riesgo de que la masa o chusma gobierne suprimiendo el derecho de las minorías (oclocracia), señalado por Polibio. Aún en el mejor de los casos, el gobierno de la buena democracia, de la mayoría con respeto de las minorías, tampoco es perfecto y hace que la democracia sea la "peor forma de Gobierno exceptuando las demás" (atribuida a Churchill), la menos mala.
} 
nomía política presentado en este artículo: Las acciones que no lleguen al nivel de las fuentes de poder y propicien reacomodos favorables a la buena gobernanza en el nivel de las prácticas sociales terminan por fortalecer la situación que pretenden superar. Los representantes de una sociedad que tengan claro esta lógica no se dejarán engañar por recetas internacionales que representan intereses particulares, resistirán la adopción nacional acrítica de políticas públicas de moda y dudarán de la retórica numérica de los reportes públicos. Por esta razón, el conocimiento y difusión del marco propuesto en este trabajo es también útil a la construcción de una buena gobernanza.

\section{Cuadro 1}

\section{Enfoques y marco conceptual propuesto para el estudio de la gobernanza}

\begin{tabular}{|c|c|c|}
\hline $\begin{array}{c}\text { Gobernanza } \\
\text { como }\end{array}$ & ¿Qué es la gobernanza? & Problemas \\
\hline $\begin{array}{l}\text { 1. Administración } \\
\text { pública tradicional } \\
\text { (APT), (autores } \\
\text { clásicos, antes de } \\
\text { 1968) }\end{array}$ & $\begin{array}{l}\text { Es el diseño e implementación } \\
\text { de políticas dirigidas a objetivos } \\
\text { específicos, políticamente definidos } \\
\text { desde el gobierno. }\end{array}$ & $\begin{array}{l}\text { Los deseos sociales son interpretados } \\
\text { e integrados en la política pública por } \\
\text { expertos al servicio del gobierno, no } \\
\text { por la participación ciudadana. }\end{array}$ \\
\hline $\begin{array}{l}\text { 2. Nueva gerencia } \\
\text { pública (NGP) } \\
\text { (Kettl 2002) }\end{array}$ & $\begin{array}{l}\text { La adopción de valores y prácticas } \\
\text { empresariales por el sector público. }\end{array}$ & $\begin{array}{l}\text { i) Concepto de gobernanza muy } \\
\text { estrecho. } \\
\text { ii) Las bases ideológicas de la NGP } \\
\text { se contraponen al papel cultural y } \\
\text { filosófico de la democracia (ver los } \\
\text { enfoques NSP y CA). }\end{array}$ \\
\hline $\begin{array}{l}\text { 3. Nuevo servicio } \\
\text { público (NSP) } \\
\text { (Denhardt y } \\
\text { Denhardt 2007) }\end{array}$ & $\begin{array}{l}\text { Son las tradiciones, instituciones y } \\
\text { procesos que determinan el ejercicio } \\
\text { delpoderenunasociedad,incluyendo } \\
\text { cómo se toman las decisiones en } \\
\text { asuntos de interés público y cómo } \\
\text { los ciudadanos participan en las } \\
\text { decisiones públicas. }\end{array}$ & $\begin{array}{l}\text { Sólo es real como planteamiento o } \\
\text { propuesta social. La multiplicación o } \\
\text { "sobrecarga" de actores no garantiza } \\
\text { que se resuelvan los problemas y } \\
\text { dificulta saber quién está a cargo o con } \\
\text { quién realizar acuerdos generales. }\end{array}$ \\
\hline
\end{tabular}




\section{Continuación cuadro 1}

\begin{tabular}{|c|c|c|}
\hline $\begin{array}{c}\text { Gobernanza } \\
\text { como }\end{array}$ & ¿Qué es la gobernanza? & Problemas \\
\hline $\begin{array}{l}\text { 4. Conjunción } \\
\text { administrativa } \\
\text { (cA) } \\
\text { (Frederickson } \\
\text { 1999) }\end{array}$ & $\begin{array}{l}\text { Es el arreglo y carácter horizontal } \\
\text { de la asociación formal e informal } \\
\text { entre los actores que representan } \\
\text { unidades, con una conducta pública } \\
\text { y administrativa interconectada. }\end{array}$ & $\begin{array}{l}\text { i) La política en cualquier } \\
\text { jurisdicción puede crear fuerzas que } \\
\text { se contrapongan a la cooperación. } \\
\text { ii) Existen riesgos de colisión de } \\
\text { personalidades entre los jefes de los } \\
\text { departamentos. } \\
\text { iii) No hay investigación satisfactoria } \\
\text { al nivel urbano, regional o } \\
\text { subnacional. }\end{array}$ \\
\hline $\begin{array}{l}\text { 5. Economía } \\
\text { política } \\
\quad(\mathrm{EP})\end{array}$ & $\begin{array}{l}\text { Son instituciones y procesos que } \\
\text { tienen que ver con el ejercicio } \\
\text { del poder en la sociedad. Los } \\
\text { determinantes de este poder se } \\
\text { remiten finalmente a la economía, } \\
\text { la fuerza, y los valores, creencias y } \\
\text { costumbres. }\end{array}$ & $\begin{array}{l}\text { Sólo hay ejemplos completos en } \\
\text { el nivel internacional (Strange y } \\
\text { Stopford 1991). }\end{array}$ \\
\hline
\end{tabular}

\section{Referencias}

Arnouts, Rikke y Bas Arts (2009). Environmental Governance Failure: The 'Dark Side' of an Essentially Optimistic Concept. En Bas Arts, Arnoud Lagendijk y Henk van Houtum, The Disoriented State: Shifts in Governmentality, Territoriality and Governance, Netherlands-Dordrecht: Springer. Environment \& Policy, Vol. 49.

Arts, Bas, Arnoud Lagendijk y Henk Van Houtum (2009). The Disoriented State: Shifts in Governmentality, Territoriality and Governance, NetherlandsDordrecht: Springer. Environment \& Policy, Vol. 49.

Behrman Jack N. y Robert E. Grosse (1990). International Business and Governments: Issues and Institutions, USA: University of South Carolina Press.

Bevir, Mark (2007a). Hollow State. En Mark Bevir (ed.), Encyclopedia of Governance, Thousand Oaks, California-London, United Kingdom-New Delhi, India: Sage, 418-419. 
(2007b). Governance. En Mark Bevir (ed.), Encyclopedia of Gover-

nance, Thousand Oaks, California-London, United Kingdom-New Delhi, India: Sage, 364-381.

Bowman, Ann. (2007). Urban Government. En Ronald K. Vogel (Editor), Handbook of Research on Urban Politics and Policy in the United States, USA: Greenwood Press.

Brewer, Thomas L. (1992). An Issue-Area Approach to the Analysis of MNE-Government Relations. Journal of International Business Studies, Vol. 23 (2) (2nd Qtr.,):295-309.

Burke, Kenneth (1945). A Grammar of Motives, California: University of California Press.

Callahan, Kathe (2007). Elements of effective governance: measurement, accountability, and participation, Boca Raton, FL.: Taylor \& Francis Group (CRC).

Dekmejian, R. Hrair y Hovann H. Simonian (2003). Troubled Waters. Geopolitics of the Caspian Region, Londres y Nueva York: I. B. Tauris.

Denhardt, Robert B. y David L. Baker (2007). Five Great Issues in Organization Theory. En Jack Rabin, W. Bartley Hildreth y Gerald J. Miller, Handbook of Public Administration, $3^{\text {rd }}$ Edition, USA: Taylor \& Francis Group.

Denhardt, Janet V. y Robert B. Denhardt (2007). The New Public Service. Serving, not Steering, Expanded Edition, USA: M. E. Sharpe.

Fenger, Menno y Victor Bekkers (2007). The Governance Concept in Public Administration. En Victor Bekkers, Geske Dijkstra, Arthur Edwards and Menno Fenger, Governance and the Democratic Deficit: Assessing the Democratic Legitimacy of Governance Practices, VT., USA: Ashgate.

Frederickson H. G. y Kevin B. Smith (2003). The Public Administration Theory Primer, USA: Westview Press. 
Frederickson, H. George (1999). The Repositioning of American Public Administration. Political Science and Politics, Vol. 32 (4), Dec., 701-711.

Gargan, John (2007). The Public Administration Community and the Search for Professionalism. En Jack Rabin, W. Bartley Hildreth y Gerald J. Miller. Handbook of Public Administration, $3^{\text {rd }}$ Edition, USA: Taylor \& Francis Group.

Giddens, A. (1976). New Rules of Sociological Method, London: Hutchinson.

Gordon, Thomas (1977). Leader Effectiveness Training: The No-Lose Way to Release the Productive Potential of People, New York: Wyden Books.

Grant, Wyn. (2003/1996). Entrada para Governability. En Iain McLean y Alistair McMillan, The Concise Oxford Dictionary of Politics, USA: The Oxford University Press.

Guess, George M. y Vache Gabrielyan (2007). Comparative and International Administration", en Jack Rabin, W. Bartley Hildreth y Gerald J. Miller. Handbook of Public Administration, $3^{\text {rd }}$ ed., USA: Taylor \& Francis Group.

Hall, John S. (2005). Governance. En Roger Caves, Encyclopedia of the City, USA y Canada: Taylor \& Francis Group, 211-212.

Hewitt de Alcántara, Cynthia. (1998). Uses and abuses of the concept of governance. International Social Science Journal, Vol. 50, Issue 155 (March),105113.

Hirst, P. (2000). Democracy and Governance. En J. Pierre (Ed.), Debating Governance. Authority, Steering and Democracy, Oxford: Oxford University Press, 13-36.

Holden, Matthew Jr. (1964). The Governance of the Metropolis as a Problem in Diplomacy. The Journal of Politics, Vol. 26, No. 3 (Aug.), 627-647.

Huff, Richard F. (2007). Governmentality. En Mark Bevir (ed.), Encyclopedia of Governance, Thousand Oaks, California-London, United Kingdom-New Delhi, India: Sage, 389-391. 
Galbraith, J. K. (1985). La anatomía del poder. 2a. ed., España: Plaza-Janes.

Gardner, John W. (1990). On Leadership, USA: The Free Press.

Kaufmann, Daniel, Aart Kraay y Massimo Mastruzzi (2009). Governance Matters VIII. Aggregate and Individual Governance Indicators, 1996-2008. Policy Research Working Paper 4978, The World Bank, Development Research Group, Macroeconomics and Growth Team (June). Disponible en: http://papers.ssrn.com/sol3/papers.cfm?abstract_id=1424591 (08/Sep/09).

Kettl, Donald F. (2002). The Transformation of Governance: Public Administration for Twenty-First Century America, USA: The Johns Hopkins University Press.

Lawton, T. C. (1996). The Creation and Control of EC Industrial Policy Lesson from the Electronics Sector (consultado en línea febrero 10/2001).

Lagendijk, Arnoud, Bas Arts y Henk van Houtum (2009). Shifts in Governmentality, Territoriality and Governance: An Introduction. En Bas Arts, Arnoud Lagendijk y Henk van Houtum, The Disoriented State: Shifts in Governmentality, Territoriality and Governance, Netherlands-Dordrecht: Springer. Environment \& Policy, Vol. 49.

Maheshwari, Shriram (2002). A Dictionary of Public Administration. New Delhi: Orient Longman.

Matkin, David S. y H. G. Frederickson (2009). Metropolitan Governance: Institutional Roles and Interjurisdictional Cooperation. Journal of Urban Affairs, Vol. 31 (1), 45-66.

Meek, Jack W. (2003). Policy Networks. En Jack Rabin, Encyclopedia of Public Administration and Public Policy, USA: Marcel Dekker (March).

Meijers, Evert Jan (2007). Synergy in Polycentric Urban Regions: Complementarity, Organising Capacity and Critical Mass, The Netherlands: IOS Press BV.

Nye, Joseph S. (2008). The Powers to Lead, USA: Oxford University Press. 
(2004). Soft Power, USA: Public Affairs (Perseus Books Group)

(1990). Bound to Lead: The Changing Nature of American Power, New York: Basic Books.

O'Boyle, C. A. (2004). Quality of Life: Assessment in Health Settings. En International Encyclopedia of the Social \& Behavioral Sciences, 12628-12631.

Peters, B. G. (2001). The Future of Governing, $2^{\text {nd }}$ ed., Lawrence, KS: University Press of Kansas.

Peters, B. G. (2007). Measurement of Governance. En Mark Bevir (ed.), Encyclopedia of Governance, Thousand Oaks, California-London, United KingdomNew Delhi, India: Sage, 364-381.

Peters, B. G. y J. Pierre (1998). Governance without Government: Rethinking Public Administration? Journal of Public Administration Research and Theory, 8, 223-243.

Pfeffer, Jeffrey (1981). Understanding the Role of Power in Decision Making. En Shafritz, Jay M. y J. Steven Ott, Classics of Organizational Theory, $4^{\text {th }}$ ed., 1987, USA: Wadsworth.

Pierre, Jon y B. Guy Peters (2005). Governing Complex Societies. Trajectories and Scenarios, New York: Palgrave MacMillan.

Rhodes, R. A. W. (2007). Understanding Governance: Ten Years On. Organization Studies 28(08), 1243-1264.

Rhodes, R. A. W. (2000). Governance and public administration. En J. Pierre (Ed.), Debating Governance: Authority Steering and Democracy, Oxford: Oxford University Press, 54-90.

Rosenau, J. N. y E. O. Czempeil (1992). Governance without Government: Order and Change in World Politics, Cambridge: Cambridge University Press.

Strange, Susan (1988). States and Markets, UK: Blackwell Publishers. 
(1996). The Retreat of the State, UK: Cambridge University Press.

y J. Stopford (1991). Rival States, Rival Firms, UK: Cambridge University Press.

Stiglitz, Joseph E. (2003). El malestar en la globalización, México: Tauros.

Terry, Larry D. (2006). The Thinning of Administrative Institutions. En David H. Rosenbloom y Howard E. McCurdy (editors), Revisiting Waldo's Administrative State: Constancy and Change in Public Administration, Washington, D.C.:Georgetown University Press, 109-128.

Van Kersbergen, K. y F. Van Waarden (2004). 'Governance' as a bridge between disciplines: cross-disciplinary inspiration regarding shifts in governance and problems of governability, accountability and legitimacy. European Journal of Political Research, 43(2), 143-172.

Wamsley, Gary L. y James F. Wolf (1996). Refounding Democratic Public Administration. Modern Paradoxes, Postmodern Challenges, USA: Sage.

Weber, Max (1957). The Concept of Power, en David L. Sills y Robert K. Merton (Eds.), International Encyclopedia of the Social Sciences. Social Sciences Quotations, 1991, Vol. 19, NY: MacMillan.

Weiss, Stephen E. (1990). The Long Path to the IBM-Mexico Agreement: An Analysis of the Microcomputer Investment Negotiations, 1983-86. Journal of International Business Studies, Vol. 21.

Whelan, Robert K. (2007). A History of the Conduct of Inquiry in Public Administration. En Jack Rabin, W. Bartley Hildreth y Gerald J. Miller, Handbook of Public Administration, $3^{\text {rd }}$ ed., USA: Taylor \& Francis Group.

Wright, Deil S. y Carl W. Stenberg (2007). Federalism, Intergovernmental Relations, and Intergovernmental Management: The Origins, Emergence, and Maturity of Three Concepts across Two Centuries of Organizing Power by Area and by Function. En Jack Rabin, W. Bartley Hildreth y Gerald J. Miller, Handbook of Public Administration, $3^{\text {rd }}$ ed., USA: Taylor \& Francis Group. (A) 
